\title{
Homotopy normal maps
}

\author{
MATAN PREZMA
}

\begin{abstract}
A group property made homotopical is a property of the corresponding classifying space. This train of thought can lead to a homotopical definition of normal maps between topological groups (or loop spaces).

In this paper we deal with such maps, called homotopy normal maps, which are topological group maps $N \rightarrow G$ being "normal" in that they induce a compatible topological group structure on the homotopy quotient $G / / N:=E N \times_{N} G$. We develop the notion of homotopy normality and its basic properties and show it is invariant under homotopy monoidal endofunctors of topological spaces, eg localizations and completions. In the course of characterizing normality, we define a notion of a homotopy action of a loop space on a space phrased in terms of Segal's 1-fold delooping machine. Homotopy actions are "flexible" in the sense they are invariant under homotopy monoidal functors, but can also rigidify to (strict) group actions.
\end{abstract}

55P35, 18D10; 18G55, 55U10, 55U15, 55U30, 55U35

\section{Introduction}

Homotopy normality is an attempt to derive a homotopical analogue for the inclusion of a normal subgroup via classifying spaces. An inclusion of topological groups $N \hookrightarrow G$ is the inclusion of a normal subgroup if and only if it is the kernel inclusion of some group map $G \rightarrow H$. Since any map is, up to homotopy, an inclusion, one needs to consider all group maps $N \rightarrow G$. Such a map should then be "homotopy normal" if $B N \rightarrow B G$ is the map from the homotopy fiber to the total space for some map $B G \rightarrow W$. There is another angle from which this notion makes sense. To every group map $N \rightarrow G$, one can associate the Borel construction $E N \times{ }_{N} G=: G / / N$, which is the "correct" quotient in the homotopical world. We note that such an extension $B G \rightarrow W$ induces a loop space structure on $G / / N$, and a loop map structure (up to map equivalence) on $G \rightarrow G / / N$, providing a second analogy to the group theoretic notion: a group inclusion $N \hookrightarrow G$ is the inclusion of a normal subgroup if and only if $G / N$ admits a group structure for which the natural quotient map $G \rightarrow G / N$ is a group map. 
Let $f: X \rightarrow Y$ be a pointed map of connected spaces. Consider the Puppe-Nomura sequence [16]

$$
\Omega X \rightarrow \Omega Y \rightarrow \Omega Y / / \Omega X \rightarrow X \rightarrow Y,
$$

where we denote $\Omega Y / / \Omega X:=\operatorname{hfib}(f)$.

The following is essentially taken from Farjoun and Hess [9, Section 5].

Definition 1.1 A loop map $\Omega f: \Omega X \rightarrow \Omega Y$ is homotopy normal if there exist a connected space $W$ with a map $\pi: Y \rightarrow W$, so that

$$
X \stackrel{f}{\longrightarrow} Y \stackrel{\pi}{\longrightarrow} W
$$

is a homotopy fibration sequence. The map $\pi: Y \rightarrow W$ is called a normal structure.

Remarks 1.2 (a) We see that a loop map $\Omega f: \Omega X \rightarrow \Omega Y$ is homotopy normal if and only if $f: X \rightarrow Y$ admits a structure of a homotopy principal fibration, ie equivalent to a principal fibration. In particular, the homotopy fiber of such a loop map has the structure of a double loop space.

(b) If $\Omega f: \Omega X \rightarrow \Omega Y$ is homotopy normal, the group map $\pi_{0}(\Omega f): \pi_{0}(\Omega X) \rightarrow$ $\pi_{0}(\Omega Y)$ is normal in the sense of [9], ie underlies a crossed module structure on the corresponding groups. Whitehead showed [23] that crossed modules correspond to connected 2-types. We note that if a discrete group map $N \rightarrow G$ is normal (in the sense of [9]) and $B G \rightarrow W$ its normal structure then $W$ is the corresponding connected 2-type.

Example 1 If $F \rightarrow E \rightarrow B$ is a fibration sequence, the map $\pi_{1} F \rightarrow \pi_{1} E$ is a homotopy normal map of discrete groups. It is also true that any homotopy normal map of discrete groups is of this form (see Brown, Higgins and Sivera [3, Section 2.6] and Loday [13, Corollary 1.5]).

Example 2 Any double loop map $\Omega^{2} f: \Omega^{2} X \rightarrow \Omega^{2} Y$ where $X, Y$ are simply connected spaces is homotopy normal: take $W=\mathrm{hfib}(X \rightarrow Y) ; W$ is then a connected space which extends the Puppe-Nomura sequence.

Example 3 Let $F$ be a pointed connected space. Then the universal fibration in Gottlieb [11], $F \rightarrow \operatorname{Baut}_{*}(F) \rightarrow \operatorname{Baut}(F)$ induces a homotopy normal map $\Omega F \rightarrow$ $\Omega \mathrm{Baut}_{*}(F)$. This map may be viewed as a universally initial homotopy normal map 
in the following sense: for every homotopy normal map $\Omega F \rightarrow \Omega X$ there exist a loop map $\Omega X \rightarrow \Omega \operatorname{Baut}_{*}(F)$ and a homotopy commutative triangle

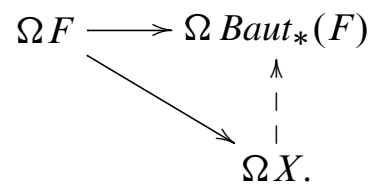

The dashed arrow is obtained as follows. Assume $F \rightarrow X \rightarrow W$ is a homotopy fibration sequence giving a normal structure on $\Omega F \rightarrow \Omega X$. By [11], there exists a "classifying map" $c: W \rightarrow \operatorname{Baut}(F)$ such that $X \rightarrow W$ is obtained as a homotopy pullback

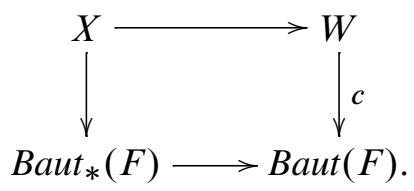

This can be extended to a homotopy commutative diagram

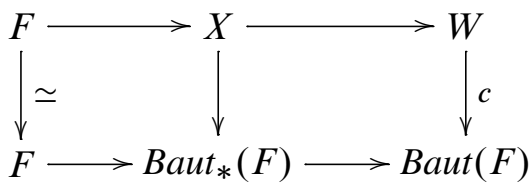

and looping down $X \rightarrow \operatorname{Baut}_{*}(F)$ gives the desired map.

\section{Main results}

Given a group map $N \rightarrow G$, each level of the bar construction $\operatorname{Bar}_{\bullet}(G, N)=$ $\left\{G \times N^{k}\right\}_{k \geq 0}$ (see May [14, Section 7]) admits an action of $G$, namely the one induced from the group inclusions $s_{0}: G \rightarrow G \times N, s_{1} s_{0}: G \rightarrow G \times N^{2}$, etc. Similarly, in any simplicial group $\Gamma_{\bullet}, \Gamma_{0}$ acts on each level via degeneracies (as above) and endows $\Gamma_{\bullet}$ with a structure of $\Gamma_{0}$-simplicial set.

The following is the main theorem in [9, Section 4], rephrased.

Theorem 1.3 A map of discrete groups $f: N \rightarrow G$ is homotopy normal if and only if there exists a simplicial group $\Gamma_{\bullet}$, with an isomorphism $\Gamma_{0} \cong G$ which extends to a $G$-equivariant isomorphism of simplicial sets

$$
\operatorname{Bar}_{\bullet}(G, N) \rightarrow \Gamma_{\bullet}
$$


The main goal of this work is to describe a generalization of Theorem 1.3 that characterizes all normal maps $\Omega X \rightarrow \Omega Y$. Our strategy is as follows.

In Section 3 we define a homotopical analogue to the bar construction $\operatorname{Bar}_{\bullet}(\Omega Y, \Omega X)$ in the case of loop maps $\Omega X \rightarrow \Omega Y$. In the degenerate case of $\Omega Y \simeq *, \operatorname{Bar}_{\bullet}(*, \Omega X)=$ $\operatorname{Bar}_{\bullet}(\Omega X)$, and one recovers Segal's 1 -fold delooping machine (Definition 2.2) for $\Omega X$.

Next, in Section 5 we define the notion of a homotopy action of a loop space on a space. We study its basic properties and establish a weak equivalence between the category of homotopy actions of a fixed loop space and the category of spaces with an action of a fixed topological group. The simplicial space $\operatorname{Bar} \bullet(\Omega Y, \Omega X)$ admits a canonical homotopy action of $\Omega Y$. A homotopy action of $\Omega Y$ is also defined for any simplicial loop space $\Gamma_{\bullet}$ satisfying $\Gamma_{0} \simeq \Omega Y$. Using this setup we can state a homotopical analogue of Theorem 1.3.

Theorem A A loop map $\Omega f: \Omega X \rightarrow \Omega Y$ is homotopy normal if and only if there exists a simplicial loop space $\Gamma_{\text {• with }} \Gamma_{0} \simeq \Omega Y$ (as loop spaces), and such that the canonical homotopy actions of $\Omega Y$ on $\Gamma_{\bullet}$ and on $\operatorname{Bar}_{\bullet}(\Omega Y, \Omega X)$ are weakly equivalent.

As often happens, Theorem 1.3 is a special case of Theorem A in that it is precisely its $\pi_{0}$ statement. One consequence of Theorem A is the fact that homotopy normal maps are invariant under homotopy monoidal functors.

Definition 1.4 A functor $L: T o p \rightarrow T o p$ is called a homotopy monoidal (HM) functor if it preserves homotopy equivalences, contractible spaces and finite products up to homotopy. The last condition can also be formulated as follows: for every pair of spaces $X, Y$, the canonical map $L(X \times Y) \stackrel{\widetilde{\Im}}{\rightarrow} L X \times L Y$ is a homotopy equivalence.

Let $\mathrm{L}$ be an HM functor and $\Omega f: \Omega X \rightarrow \Omega Y$ a loop map. It is implicit in Bousfield [2] and Farjoun [7] and can be proved also by using the delooping theorem of Segal [20] that $L(\Omega X)$ always has the homotopy type of a loop space and $L(\Omega f)$ is always equivalent to a loop map.

Remark 1.5 Although HM functors preserve the property of having (the homotopy type of) a loop space, they do not commute with the functor $\Omega$ : $T o p_{*} \rightarrow T o p_{*}$.

Using the fact that homotopy actions of loop spaces can be described in terms of maps between finite products of spaces we show that HM functors preserve homotopy normality. 
Theorem B Let $\Omega f: \Omega X \rightarrow \Omega Y$ be a homotopy normal map. If $L:$ Top $\rightarrow$ Top is an $H M$ functor, then $L(\Omega f): L \Omega X \rightarrow L \Omega Y$ is a homotopy normal map.

This, in turn, gives an immediate proof of a theorem due to Dwyer and Farjoun [5, Section 3] which we restate.

Theorem C Let $f: X \rightarrow Y$ be a map of pointed connected spaces and $p: E \rightarrow B$ be a homotopy principal fibration of connected spaces. If $L_{\Sigma f}$ is the localization functor by $\Sigma f: \Sigma X \rightarrow \Sigma Y$, then $L_{\Sigma f}(p): L_{\Sigma f} E \rightarrow L_{\Sigma f} B$ is a homotopy principal fibration.

Remark 1.6 In what follows, we use $L$ to denote an arbitrary HM functor. The notation $L$ reflects the special case of localization by a map.

Refer to related work of Farjoun and Hess [8] on homotopy (co)normal structures in a category with a class of weak equivalences and some additional structure, called a twisted homotopical category.

Acknowledgements This paper is based on the author's $\mathrm{PhD}$ thesis at the Hebrew University of Jerusalem. The author would like to express deep thanks to his advisor, Emmanuel Dror Farjoun for his continuous guidance, discussions and encouragement. The author would also like to thank the Hebrew University of Jerusalem for support of his studies. Special thanks are extended to David Blanc and James Stasheff for helpful suggestions and conversations.

\section{Preliminaries}

Throughout this paper, topological spaces or spaces will mean topological spaces of the homotopy type of CW complexes. We denote the corresponding category by Top. Thus, by Whitehead's theorem, every weak equivalence is in fact a homotopy equivalence. All mapping spaces will be taken with the compact-open topology. The path space $P X$ of a pointed space $X$ is the space of maps $\{\alpha: I \rightarrow X \mid \alpha(0)=*\}$; a loop space is understood to be a space of the form $\Omega X:=\{\alpha: I \rightarrow X \mid \alpha(0)=*=\alpha(1)\}$, where $X$ is a pointed connected space and a loop map is a map of the form $\Omega f: \Omega X \rightarrow \Omega Y$ where $f: X \rightarrow Y$ is a pointed map. The following is a well-known fact, essentially contained in Kan [12] and Milnor [15]. 
Theorem 2.1 If $X$ is a (pointed) connected space, then there exists a topological group $G$, with $X \stackrel{\simeq}{\rightarrow} B G$. Moreover, one can construct $G$ functorially in $X$, ie if $\Omega f: \Omega X \rightarrow \Omega Y$ is a loop map, there is a commutative diagram

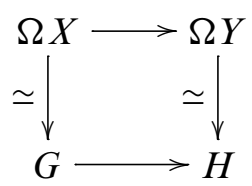

with the vertical arrows being homotopy equivalences, and the bottom arrow being a topological group map.

A map $E \rightarrow B$ is a (Serre) fibration if it has the right lifting property with respect to all inclusions of the form $D^{n} \hookrightarrow D^{n} \times I$ that include the $n$-disc $D^{n}$ as $D^{n} \times\{0\}$. A fibration sequence is a sequence of the form $F \rightarrow E \stackrel{p}{\rightarrow} B$, where $p: E \rightarrow B$ is a fibration and either $\left(B, b_{0}\right)$ is pointed and $F=p^{-1}\left(b_{0}\right)$ or $F=p^{-1}(b)$ for some $b \in B$ and $B$ is connected. A sequence $X \rightarrow Y \rightarrow Z$ is called a homotopy fibration sequence if there is a commutative diagram

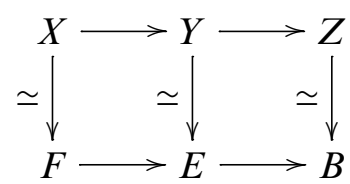

with vertical arrows being homotopy equivalences and the bottom being a fibration sequence. A homotopy fibration sequence $X \rightarrow Y \rightarrow Z$ is called a homotopy principal fibration sequence if there is a connected space $B$ and a map $Z \rightarrow B$, called the classifying map such that $Y \rightarrow Z \rightarrow B$ is a homotopy fibration sequence. In that case, $X \simeq \Omega B$ and there is a principal fibration sequence $G \rightarrow E \rightarrow E / G$, and a commutative diagram

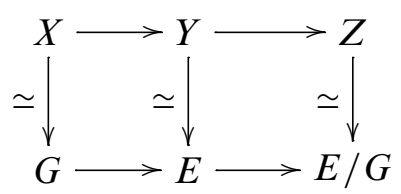

with all vertical maps being homotopy equivalences and the left vertical map being equivalent to a loop map $\Omega B \rightarrow \Omega B G$.

As usual, we denote by $\Delta$ the category of finite ordinals $[n]=(0, \ldots, n)$ with ordinal maps between them. Given a category $\mathcal{C}$, a simplicial object in $\mathcal{C}$ is a functor $\Delta^{\text {op }} \rightarrow \mathcal{C}$, and we denote it by $X_{\bullet}$ with $X_{n}$ for its value on $[n]$.

Of special importance to this paper are simplicial objects in Top, namely simplicial spaces. If $X$ is a space, we shall denote the constant simplicial space on it by $X$ 
when there is no risk of confusion. An equivalence of simplicial spaces (or: simplicial equivalence) is a simplicial map $f: X_{\bullet} \rightarrow Y_{\bullet}$ such that, $f_{n}: X_{n} \rightarrow Y_{n}$ is a homotopy equivalence for each $n$. Similarly, a (homotopy) fibration sequence of simplicial spaces is a diagram of simplicial spaces $F_{\bullet} \rightarrow E_{\bullet} \rightarrow B_{\bullet}$ which is a level-wise (homotopy) fibration sequence.

We will often use a particular class of simplicial spaces introduced in a preprint of Segal [20] and originally called "group-like special $\Delta$-spaces". Influenced by the Rezk's terminology [19], we call them reduced Segal spaces; these are defined as follows.

Definition 2.2 (cf [20]) (a) A reduced Segal space is a simplicial space $B$ • such that

(i) $B_{0} \simeq *$;

(ii) for each $n \geq 1$, the maps $p_{n}: B_{n} \rightarrow B_{1} \times \cdots \times B_{1}$ (called Segal maps) induced by the maps

$$
\begin{gathered}
i_{k}:[1] \rightarrow[n] \quad(1 \leq k \leq n) \\
0 \mapsto k-1, \quad 1 \mapsto k,
\end{gathered}
$$

are homotopy equivalences;

(iii) the monoid structure on $\pi_{0}\left(B_{1}\right)$ admits inverses (ie is a group).

(b) We say that $B_{\bullet}$ is a reduced Segal space for $\Omega X$ if it comes equipped with a homotopy equivalence $\left|B_{\bullet}\right| \stackrel{\widetilde{\sim}}{\rightarrow} X$; if $B_{\bullet}$ and $B_{\bullet}^{\prime}$ are reduced Segal spaces for $\Omega X$, a map (respectively equivalence) between them is a simplicial map (respectively equivalence) $B_{\bullet} \rightarrow B_{\bullet}^{\prime}$ which makes the triangle of loop maps below commutative.

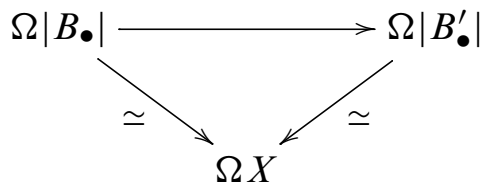

Remark 2.3 By [20, 1.5], it follows that if $B$ • is a reduced Segal space for $\Omega X$ there is a natural homotopy equivalence $B_{1} \stackrel{\widetilde{\Im}}{\rightarrow} \Omega\left|B_{\bullet}\right|$. Thus, a reduced Segal space for $\Omega Y$ can equivalently be defined as a reduced Segal space $B_{\bullet}$ equipped with a loop equivalence $B_{1} \stackrel{\simeq}{\rightarrow} \Omega X$. The diagram of Definition 2.2(b) should then be changed accordingly.

For a topological group $G$ and $a: X \times G \rightarrow X$ a right action of $G$ on a space $X$ which we denote by $x \mapsto x g$ for $x \in X$ and $g \in G$, the bar construction (cf [14, Section 7]) is the simplicial space $\operatorname{Bar}_{\bullet}(X, G)$, consisting of 
(1) for every $n \geq 0, \operatorname{Bar}_{n}(X, G):=X \times G^{n}$

together with

(2) face maps $d_{i}^{(n)} \equiv d_{i}: \operatorname{Bar}_{n}(X, G) \rightarrow \operatorname{Bar}_{n-1}(X, G)$ for every $n \geq 1$ and every $0 \leq i \leq n$ given by

$$
d_{i}:\left(x, g_{1}, \ldots, g_{n}\right) \mapsto \begin{cases}\left(x \cdot g_{1}, g_{2}, \ldots, g_{n}\right) & \text { if } i=0, \\ \left(x, g_{1}, \ldots, g_{i-1}, g_{i} \cdot g_{i+1}, g_{i+2}, \ldots, g_{n}\right) & \text { if } 1 \leq i<n, \\ \left(x, g_{1}, \ldots, g_{n-1}\right) & \text { if } i=n,\end{cases}
$$

(3) degeneracy maps $s_{i}: \operatorname{Bar}_{n}(X, G) \rightarrow \operatorname{Bar}_{n+1}(X, G)$ for every $n \geq 1$ and every $0 \leq i \leq n$ given by

$$
s_{i}:\left(x, g_{1}, \ldots, g_{n}\right) \mapsto\left(x, g_{1}, \ldots, g_{i}, e, g_{i+1}, \ldots, g_{n}\right) .
$$

\section{The homotopy power of a map}

Given a fibration $p: E \rightarrow B$, one can define a simplicial space $P o w_{\bullet}(E \rightarrow B)$, called the power of $p$, by $\operatorname{Pow}_{n}(E \rightarrow B)=E \times_{B} E \cdots \times_{B} E(n+1$ times) with face and degeneracies being the obvious projections and diagonals. In [13], it is shown that for ( $E$ nonempty and) $B$ connected, $\left|\operatorname{Pow}_{\bullet}(E \rightarrow B)\right| \simeq B$. We note that for a nonconnected space $B,\left|\operatorname{Pow}_{\bullet}(E \rightarrow B)\right|$ is homotopy equivalent to the disjoint union of connected components of $B$ intersecting the image of $p$.

Here, we wish to construct such a power space for an arbitrary map $f: X \rightarrow B$ by means of homotopy pullbacks, thus turning it to a homotopically invariant construction.

We define the $n$-th homotopy power of $f: X \rightarrow B$ to be

$$
\text { hPow }_{n}(X \rightarrow B)=\operatorname{map}\left(\begin{array}{cc}
\Delta[n]_{0} & X \\
\iota \downarrow & \downarrow \\
\Delta[n] & b
\end{array}\right)=\operatorname{holim}\left(\begin{array}{ccc}
X & X & \cdots \\
\downarrow & \downarrow \\
B
\end{array}\right),
$$

with $\iota: \Delta[n]_{0} \rightarrow \Delta[n]$ being the inclusion of the 0 -skeleton into the topological $n-$ simplex.

This clearly yields a functorial construction over $\Delta^{\mathrm{op}}$, and we define:

Definition 3.1 The homotopy power of a map $f: X \rightarrow B$, denoted $h$ Pow $_{\bullet}(X \rightarrow B)$, is the simplicial space with $\operatorname{PPow}_{n}(X \rightarrow B)$ on level $n$, and face and degeneracies given by the functorial construction above. 
Note that for a fibration $p: E \rightarrow B$ one gets an equivalence of simplicial spaces hPow. $_{\bullet}(E \rightarrow B) \simeq$ Pow $_{\bullet}(E \rightarrow B)$.

Remark 3.2 When calculating the homotopy power of a map $f: X \rightarrow B$ we will often use a slightly different but equivalent construction. We first replace $f$ by an equivalent fibration $p: E_{f} \rightarrow B$, ie one for which there is a commutative triangle

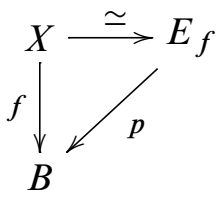

and then take the power of $p$, as in [13]. This construction is functorial as well. We also note that if $X \rightarrow B$ is a pointed map, $h \operatorname{Pow}_{\bullet}(X \rightarrow B)$ naturally becomes a pointed simplicial space.

\section{The homotopy bar construction}

Consider a topological group $G$ acting on a space $X$ and the corresponding (homotopy) principal fibration $G \rightarrow X \rightarrow X / / G$. One has the "usual" bar construction $\operatorname{Bar}_{\bullet}(X, G)=\left\{X \times G^{k}\right\}_{k \geq 0}$ with $\left|\operatorname{Bar}_{\bullet}(X, G)\right|=X / / G$. On the other hand, we can resolve $X / / G$ by taking homotopy powers of the map $q: X \rightarrow X / / G$.

Proposition 4.1 Let $G$ act on $X$ as above. Then there are simplicial equivalences

$$
\operatorname{Bar}_{\bullet}(X, G) \rightleftarrows \text { Pow. }_{\bullet}(X \rightarrow X / / G) \text {. }
$$

Proof Replacing $q: X \rightarrow E G \times_{G} X$ by the fibration $p: E G \times X \rightarrow E G \times_{G} X$ and taking the pullback, we get $h \operatorname{Pow}_{1}(X \rightarrow X / / G)=(E G \times X) \times_{X / / G}(E G \times X) \cong$ $E G \times G \times X$, since $E G \times X$ is a free $G$-space. In general,

$$
\text { hPow }_{n}(X \rightarrow X / / G)=(E G \times X) \times_{X / / G} \cdots \times_{X / / G}(E G \times X) \cong E G \times X \times G^{n},
$$

and the obvious map $E G \times X \times G^{n} \rightarrow X \times G^{n}$ defines a simplicial equivalence $h_{P o w}(X \rightarrow X / / G) \rightarrow \operatorname{Bar}_{\bullet}(X, G)$. Taking (for example) Milnor's join construction, we have a natural base point for $E G$ and hence a canonical map $X \times G^{n} \rightarrow E G \times X \times G^{n}$, which in turn defines another simplicial equivalence.

In light of the last proposition, we define:

Definition 4.2 Given a (homotopy) principal fibration sequence $\Omega Y \rightarrow X \stackrel{q}{\rightarrow} Q$, the homotopy bar construction $\operatorname{Bar}_{\bullet}(X, \Omega Y)$ is the homotopy power $h \operatorname{Pow}_{\bullet}(X \rightarrow Q)$. 
Remark 4.3 In the case of a loop map $\Omega f: \Omega Y \rightarrow \Omega Z, \operatorname{Bar}_{\bullet}(\Omega Z, \Omega Y)$ is the homotopy power of the map $q: \Omega Z \rightarrow \Omega Z / / \Omega Y:=\mathrm{hfib}(f)$. If $\Omega Z \simeq *, \operatorname{Bar}_{\bullet}(*, \Omega Y)$ becomes the power of the map $P Y \rightarrow Y$ which is a reduced Segal space for $\Omega Y$. Put differently, one can recover Segal's delooping machine by using homotopy powers.

It is useful to have the following property.

Proposition 4.4 Let $f: X \rightarrow B$ be any pointed map. The canonical map induces an equivalence of simplicial spaces $\Omega\left(h\right.$ Pow $\left._{\bullet}(X \rightarrow B)\right) \simeq h_{\text {Pow }}(\Omega X \rightarrow \Omega B)$.

The proof is essentially the fact that given a pointed diagram $A \rightarrow X \leftarrow Y$, we have a weak equivalence $\Omega \operatorname{holim}(A \rightarrow X \leftarrow Y) \simeq \operatorname{holim}(\Omega A \rightarrow \Omega X \leftarrow \Omega Y)$.

\subsection{From homotopy normality to a simplicial loop space structure on the homotopy bar construction}

Let $\Omega f: \Omega X \rightarrow \Omega Y$ be a homotopy normal map. We form the Puppe-Nomura sequence

$$
\Omega X \stackrel{\Omega f}{\longrightarrow} \Omega Y \stackrel{q}{\longrightarrow} \Omega Y / / \Omega X \longrightarrow X \longrightarrow Y \stackrel{\pi}{\longrightarrow} W .
$$

Then by [16] there is a commutative triangle in which the vertical arrow is a homotopy equivalence

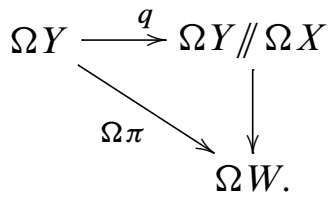

Passing to (homotopy) powers, we get an equivalence of simplicial spaces

$$
\text { hPow. }_{\bullet}(\Omega Y \rightarrow \Omega W) \simeq \text { PPow. }_{\bullet}(\Omega Y \rightarrow \Omega Y / / \Omega X)
$$

and, by Proposition 4.4, an equivalence of simplicial spaces

$$
\Omega\left(h \text { Pow }_{\bullet}(Y \rightarrow W)\right) \simeq \text { Pow }_{\bullet}(\Omega Y \rightarrow \Omega Y / / \Omega X) .
$$

Using the argument above and Definition 4.2 we have just proved the following result.

Theorem 4.5 If $\Omega f: \Omega X \rightarrow \Omega Y$ is homotopy normal, there are natural simplicial equivalences $\operatorname{Bar}_{\bullet}(\Omega Y, \Omega X) \rightleftarrows \Omega\left(\operatorname{hPow}_{\bullet}(Y \rightarrow W)\right)$.

Notation 4.6 (cf Theorem 4.5) (1) For a homotopy normal map $\Omega f: \Omega X \rightarrow \Omega Y$ and a given normal structure $\pi: Y \rightarrow W$, we denote by $Q$ • the simplicial loop space $\Omega\left(\right.$ Prow $\left._{\bullet}(Y \rightarrow W)\right)$. 
(2) The equivalences given in Theorem 4.5 will be denoted

$$
\epsilon: \operatorname{Bar}_{\bullet}(\Omega Y, \Omega X) \rightleftarrows Q \bullet: \eta .
$$

Remark 4.7 Notice that the maps

$$
\epsilon_{0}: \Omega Y \rightleftarrows Q_{0}: \eta_{0}
$$

are loop maps by construction, but for $n \geq 1$, the maps

$$
\epsilon_{n}: \operatorname{Bar}_{n}(\Omega Y, \Omega X) \rightleftarrows Q_{n}: \eta_{n}
$$

need not be loop maps. This means that we have, in general, two different loop space structures on $\Omega Y \times(\Omega X)^{n}$. The nontrivial one is given by the equivalence $\operatorname{Bar}_{n}(\Omega Y, \Omega X) \simeq Q_{n}$.

\section{Homotopy actions}

By Remarks 1.2(a) a homotopy normal map is a loop map with its underlying map having the structure of a principal fibration (of connected spaces). Furthermore, Theorem 1.3 involves (strict) group actions. Hence, characterization and invariance of homotopy normal maps under HM functors should include characterization and invariance of group actions "up to homotopy" to some extent. Given an action of a topological group $G$ on a space $X$ and an HM functor $L: T o p \rightarrow T o p$, we would like to construct a canonical "action" of $L G$ (not a group, not a loop space) on $L X$. In other words, we would like to have a homotopical notion of an action of (a space of the homotopy type of) a loop space on a space, invariant under HM functors. One approach we wish to refer the reader to is that of $A_{\infty}$-actions introduced by Nowlan [17] and recently used by Stasheff [21]. For our purpose, we could not use $A_{\infty}$-actions since it is not clear they are invariant under HM functors. As demonstrated in Section 5.2, homotopy actions can be rigidified into (strict) group actions. This rigidification gives in fact a "proxy action" on $X$ in the sense of Dwyer and Wilkinson [6] so all the homotopically-invariant information (eg homotopy fixed points) is preserved. Homotopy actions have more flexibility than proxy actions since the object which "acts" need not be a topological group but rather a loop space.

\subsection{Definition and basic properties}

If a topological group $G$ acts on a space $X$, one has a simplicial fibration sequence of the form $X \rightarrow \operatorname{Bar}_{\bullet}(X, G) \rightarrow B_{\bullet} G$, where the maps $X \rightarrow \operatorname{Bar}_{n}(X, G)$ and $\operatorname{Bar}_{n}(X, G) \rightarrow B_{n} G$ are given by $s_{n} \cdots s_{0}$ and projection respectively. 
Under realization, this becomes a (homotopy) fibration sequence $X \rightarrow X / / G \rightarrow B G$ with a connected base space, ie an "action up to homotopy" in the sense of Dror, Dwyer and Kan [4]. The above simplicial fibration sequence is trivial in each level $X \rightarrow X \times G^{n} \rightarrow G^{n}$, and hence constitutes a useful resolution. We note also that for all $n$, the map $d_{1} d_{2} \cdots d_{n}: \operatorname{Bar}_{n}(X, G) \rightarrow \operatorname{Bar}_{0}(X, G)$ is the projection on $\mathrm{X}$ and the map $d_{0} d_{0} \cdots d_{0}: \operatorname{Bar}_{n}(X, G) \rightarrow \operatorname{Bar}_{0}(X, G)$ is given by $\left(x, g_{1}, \ldots, g_{n}\right) \mapsto x \cdot\left(g_{1} \cdots g_{n}\right)$.

As we saw, the simplicial spaces $\operatorname{Bar} \bullet(X, G)$ and $B_{\bullet} G$ can be relaxed to their "homotopy versions", namely $\operatorname{Bar}_{\bullet}(X, \Omega Y)$ and $\operatorname{Bar}_{\bullet}(*, \Omega Y)$ (which is a reduced Segal space for $\Omega Y$ when $B G \simeq Y$ ).

Definition 5.1 We say that a space $S$ of the homotopy type of a loop space, homotopy acts on a space $X$, if there exist a simplicial map

$$
A \bullet \stackrel{\pi}{\longrightarrow} B \bullet
$$

such that

(1) $A_{0} \simeq X$;

(2) $B_{\bullet}$ is a reduced Segal space for $S$;

(3) for every $n$, the maps

$$
A_{n} \underset{d_{0} \cdots d_{0} \times \pi_{n}}{\stackrel{d_{1} \cdots d_{n} \times \pi_{n}}{\longrightarrow}} A_{0} \times B_{n}
$$

are homotopy equivalences.

Maps are defined as follows.

Definition 5.2 Given two homotopy actions of $S$ on $X$ and on $X^{\prime}$, represented by $A_{\bullet} \rightarrow B_{\bullet}$ and $A_{\bullet}^{\prime} \rightarrow B_{\bullet}^{\prime}$ respectively, a map between them is a commutative square

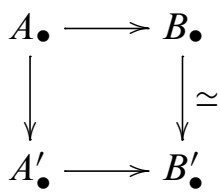

such that the map $B_{\bullet} \rightarrow B_{\bullet}^{\prime}$ is an equivalence of reduced Segal spaces (see Definition 2.2).

Notation 5.3 We denote by $T o p^{h \Omega Y}$ the category of homotopy actions of (spaces of the homotopy type of) $\Omega Y$ on spaces. 
Remark 5.4 If $S \rightarrow S^{\prime}$ is a loop equivalence and $S$ homotopy acts on $X$, then $S^{\prime}$ homotopy acts on $X$, since a reduced Segal space $B \bullet$ for $S$ induces a reduced Segal

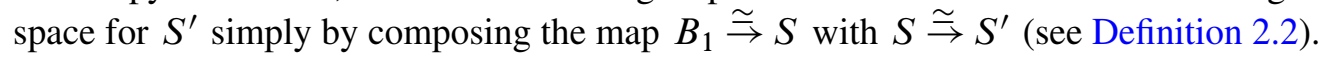

We will need a generalization of Definition 5.1 as follows.

Definition 5.5 A homotopy action of $\Omega Y$ on a simplicial space $X \bullet$ is a map of bisimplicial spaces $A_{\bullet \bullet} \rightarrow B_{\bullet \bullet}$ such that for each $n, A_{\bullet n} \rightarrow B_{\bullet n}$ is a homotopy action of $\Omega Y$ on $X_{n}$ and for every map $\theta:[n] \rightarrow[m]$ in $\Delta, \theta^{*}: B_{\bullet m} \rightarrow B_{\bullet n}$ is an equivalence of reduced Segal spaces for $\Omega Y$; maps and equivalences are defined in the obvious way.

Observation 5.6 If a topological group $G$ acts on a space $X$, the simplicial map $p: \operatorname{Bar}_{\bullet}(X, G) \rightarrow B_{\bullet}(G)$ is a homotopy action of $G$ on $X$. To see this, note that $B_{\bullet}(G)$ is a reduced Segal space for $G$ and the maps $\left(d_{1} \cdots d_{n}\right) \times p_{n}: \operatorname{Bar}_{n}(X, G) \rightarrow$ $\operatorname{Bar}_{0}(X, G) \times B_{n}(G)$ are the identity maps $X \times G^{n} \rightarrow X \times G^{n}$. One can verify that the maps $\left(d_{0} \cdots d_{0}\right) \times p_{n}: \operatorname{Bar}_{n}(X, G) \rightarrow \operatorname{Bar}_{0}(X, G) \times B_{n}(G)$, ie the action of $G^{n}$ on $X$ (arising from multiplying $n$ elements in $G$ and then act on $X$ ) multiplied by the projection $p_{n}$, are homeomorphisms.

Nowlan [17] defined an action of an $A_{\infty}$-space on a topological space. The difference between this approach and ours is essentially the difference between the approaches of Stasheff [22] and Segal [20] to the characterization of loop spaces.

It is commonly said that in every fibration sequence, the loop space of the base "acts" on the fiber. We wish to demonstrate how a homotopy action interprets this statement.

Theorem 5.7 Given a fibration sequence $F \stackrel{i}{\rightarrow} E \stackrel{p}{\rightarrow} B$ with $B$ pointed connected, there is a homotopy action of $\Omega B$ on $F$, represented by $\pi: A_{\bullet} \rightarrow B_{\bullet}$, such that the map $|\pi|:\left|A_{\bullet}\right| \rightarrow\left|B_{\bullet}\right|$ is equivalent to $p: E \rightarrow B$.

Proof Consider the commutative square

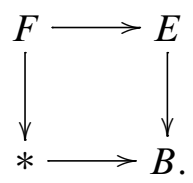

Taking homotopy powers in each row produces a simplicial map

$$
\pi: A_{\bullet}:=\operatorname{hPow}_{\bullet}(F \rightarrow E) \rightarrow \operatorname{hPow}_{\bullet}(* \rightarrow B)=: B_{\bullet} .
$$

By Remark 4.3, $B_{\bullet}$ is a reduced Segal space and thus $\left|B_{\bullet}\right| \simeq B$. Since $B$ is connected, it follows from Section 3 that $\left|A_{\bullet}\right| \simeq E$. To see that $\pi: A_{\bullet} \rightarrow B_{\bullet}$ is a homotopy 
action, we first replace $i: F \rightarrow E$ and $* \rightarrow B$ by equivalent fibrations ev ev $_{1}: F_{i} \rightarrow E$ and $\mathrm{ev}_{1}: P B \rightarrow B$, where $P B$ is the path space and $F_{i} \subseteq F \times E^{I}$ is the space $\{(f, \alpha) \mid \alpha(0)=i(f)\}$. Taking $\pi_{0}: F_{i} \rightarrow P B$ to be $\pi_{0}(f, \alpha)=p \circ \alpha$ we obtain the commutative square

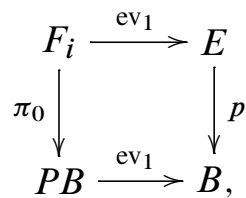

and taking powers (ie fiber products) of the rows, we obtain a simplicial map we denote as $\pi: A \bullet \rightarrow B \bullet$.

Let us show that the maps

$$
A_{1} \underset{d_{0} \cdots d_{0} \times \pi_{1}}{\stackrel{d_{1} \cdots d_{n} \times \pi_{1}}{\longrightarrow}} A_{0} \times B_{1}
$$

are homotopy equivalences. We have a commutative cube

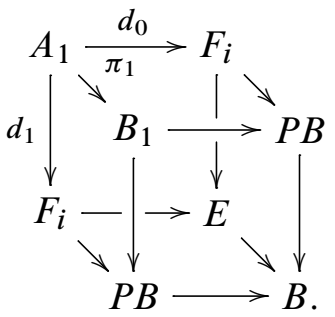

We want to show that the left-hand and upper faces are homotopy cartesian squares, which follows directly from the cartesian-ness of the lower, right-hand and outer faces using the fact that a square is cartesian if and only if the comparison map between homotopy fibers of rows/columns is a homotopy equivalence; see Goodwillie [10, 1.18].

One proceeds similarly to show that the maps $\left(d_{0} \cdots d_{0}\right) \times \pi_{n}$ and $\left(d_{1} \cdots d_{n}\right) \times \pi_{n}$ $(n>1)$ are homotopy equivalence. Thus, $\pi: A_{\bullet} \rightarrow B_{\bullet}$ is a homotopy action.

Lastly, since the equivalences $\mid$ Pow $_{\bullet}\left(F_{i} \rightarrow E\right) \mid \simeq E$ and $\left|P_{\bullet} w_{\bullet}(P B \rightarrow B)\right| \simeq B$ are natural, and in light of (*) the map $|\pi|:\left|A_{\bullet}\right| \rightarrow\left|B_{\bullet}\right|$ is equivalent to $p: E \rightarrow B$.

The importance of Theorem 5.7 can be seen, for example, from the fact that it allows one to classify fibrations using homotopy actions.

Homotopy actions arise in our context in the following form. 
Corollary 5.8 If $\Omega f: \Omega X \rightarrow \Omega Y$ is a loop map, then $\Omega f$ induces a homotopy action of $\Omega X$ on $\Omega Y$, natural in $f$.

Proof This follows from Theorem 5.7 if we consider the homotopy fibration sequence $\Omega Y \rightarrow \Omega Y / / \Omega X \rightarrow X$. Alternatively, if we (functorially) rigidify $\Omega f: \Omega X \rightarrow \Omega Y$ to a topological group map $G \rightarrow H$ as in Theorem 2.1, then as we saw, $\operatorname{Bar}_{\bullet}(H, G) \rightarrow B \bullet G$ is a homotopy action.

Finally, let us see that homotopy actions are invariant under HM functors.

Proposition 5.9 If $A_{\bullet} \rightarrow B_{\bullet}$ is a homotopy action of $\Omega Y$ on $X$, and $L: T o p \rightarrow T o p$ is an HM functor, then $L A \bullet \rightarrow L B \bullet$ is a homotopy action of $L \Omega Y$ on $L X$.

Proof $L B_{\bullet}$ is a reduced Segal space for $L B_{1}$. In particular, $L B_{1}$ is of the homotopy type of a loop space. Applying $L$ to the structure maps of the homotopy action yields the structure maps for $L A_{\bullet} \rightarrow L B_{\bullet}$, and $L$ preserves homotopy equivalences.

For the sake of completeness, we wish to define a map between homotopy actions of two non-homotopy equivalent loop spaces. The simplicity of the definition demonstrates the "flexibility" of homotopy actions. For example, it allows one to talk about the category of all homotopy actions.

Definition 5.10 Given two homotopy actions of $\Omega Y$ on $X$ and of $\Omega\left(Y^{\prime}\right)$ on $X^{\prime}$, represented by $A_{\bullet} \longrightarrow B_{\bullet}$ and $A_{\bullet}^{\prime} \longrightarrow B_{\bullet}^{\prime}$, a map between them is a commutative square of simplicial spaces

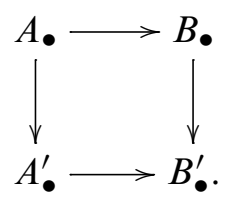

Such a map will be called an equivalence if both vertical maps are simplicial equivalences.

\subsection{A weakly inverse correspondence with group actions}

Our goal here is to establish a weakly inverse correspondence between the category $T o p_{B G}$ of spaces over $B G$ and the category $T o p^{h \Omega Y}$ of homotopy actions of $\Omega Y$ where $Y \simeq B G$. Since $T o p_{B G}$ is Quillen equivalent to the category of $G$-spaces, we obtain a correspondence between homotopy actions and group actions which may be referred to as a "rigidification" of the homotopy action. Our functors will be weak inverses in the following sense. 
Definition 5.11 Maps $f: X \rightarrow Y$ and $f^{\prime}: X^{\prime} \rightarrow Y^{\prime}$ are called weakly equivalent if there is a zigzag of commutative squares with all horizontal arrows being homotopy equivalences

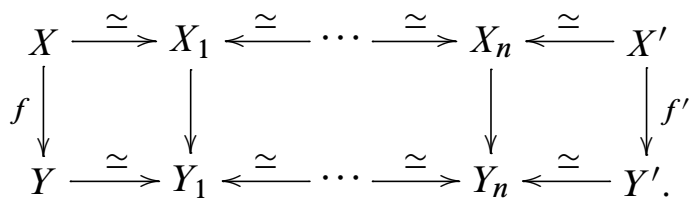

Similarly, simplicial maps $f: X_{\bullet} \rightarrow Y_{\bullet}$ and $f^{\prime}: X_{\bullet}^{\prime} \rightarrow Y_{\bullet}^{\prime}$ are called weakly equivalent if there is a zigzag of commutative squares as above, but with objects being simplicial spaces and maps being simplicial maps. The number of squares involved in such a zigzag is said to be its length. In particular, maps are called equivalent if they are weakly equivalent via a zigzag of length 1 .

Definition 5.12 Let $G$ be a topological group, $\Omega Y$ a loop space and $Y \rightarrow B G$ a fixed homotopy equivalence.

(1) The functor $\mathcal{P}: T_{0} p_{B G} \rightarrow \operatorname{Top}^{h \Omega Y}$ is defined as follows. Given a map $E \rightarrow B G$, let $X$ be its homotopy fiber. Thus, there is a commutative square

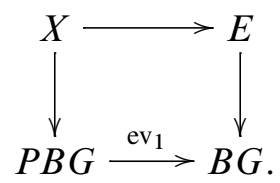

Then $\mathcal{P}(E \rightarrow B G)$ is the map hPow $(X \rightarrow E) \rightarrow h$ Pow $_{\bullet}(P B G \rightarrow B G)$, which is a homotopy action of $\Omega Y$ by Theorem 5.7.

(2) The functor $\mathcal{R}: T o p^{h \Omega Y} \rightarrow T_{o p} p_{B G}$ is defined as follows. Given a homotopy action $\pi: A_{\bullet} \rightarrow B_{\bullet}$ of $\Omega Y$ on $X, \mathcal{R}\left(A_{\bullet} \rightarrow B_{\bullet}\right)$ is the composition

$$
\left|A_{\bullet}\right| \stackrel{|\pi|}{\rightarrow}\left|B_{\bullet}\right| \stackrel{\simeq}{\rightarrow} Y \stackrel{\simeq}{\rightarrow} B G,
$$

where the second map comes from the fact that $B_{\bullet}$ is a reduced Segal space for $\Omega Y$ (see Definition 2.2).

Proposition 5.13 The functors above satisfy the following properties.

(a) If $E \rightarrow B G$ is in $T o p_{B G}$, then $\mathcal{P}(E \rightarrow B G)$ is a homotopy action of $\Omega Y$ on $X:=\operatorname{hfib}(E \rightarrow B G)$.

(b) If $\pi: A_{\bullet} \rightarrow B_{\bullet}$ is a homotopy action of $\Omega Y$ on $X$, then $\mathcal{R}\left(A_{\bullet} \rightarrow B_{\bullet}\right)$ is a space over $B G$ with $X$ as its homotopy fiber. 
Proof (a) This follows from Theorem 5.7.

(b) Given a homotopy action $\pi: A \bullet \rightarrow B \bullet$ of $\Omega Y$ on $X$, define a simplicial map $i: A_{0} \rightarrow A_{\bullet}$ by $i_{n}=s_{n-1} \cdots s_{0}$. Choose $b_{0} \in B_{0}$ and endow $B_{n}$ with a basepoint $s_{n-1} \cdots s_{0}\left(b_{0}\right)$. By definition, the map $\left(d_{1} \cdots d_{n}\right) \times \pi_{n}: A_{n} \rightarrow A_{0} \times B_{n}$ is a homotopy equivalence and hence the map $\pi_{n}: A_{n} \rightarrow B_{n}$ is equivalent to the trivial fibration $A_{0} \times B_{n} \rightarrow B_{n}$. We now claim that

$$
A_{0} \stackrel{i_{n}}{\rightarrow} A_{n} \stackrel{\pi_{n}}{\rightarrow} B_{n}
$$

is a homotopy fibration sequence. To see this, note that by simplicial identities, the composite

$$
A_{0} \stackrel{\left(d_{1} \cdots d_{n} \times \pi_{n}\right) \circ i_{n}}{\longrightarrow} A_{0} \times B_{n}
$$

equals $1_{A_{0}} \times\left(\pi_{n} \circ i_{n}\right)$ and, since $B_{0}$ is contractible, $\pi_{n} \circ i_{n}=s_{n-1} \cdots s_{0} \circ \pi_{0}$ is null-homotopic. Hence, $i_{n}$ is equivalent to the fiber inclusion $A_{0} \rightarrow A_{0} \times B_{n}$. It follows that the sequence $A_{0} \rightarrow A_{\bullet} \rightarrow B_{\bullet}$ is a homotopy fibration sequence in each level and so $A_{0} \rightarrow\left|A_{\bullet}\right| \rightarrow\left|B_{\bullet}\right|$ is a homotopy fibration sequence by Puppe [18]. By definition, $A_{0} \simeq X$, and we are done.

Theorem 5.14 The functors $\mathcal{R}$ : Top ${ }^{h \Omega Y} \rightleftarrows T_{\text {Top }}$ : $: \mathcal{P}$ of Definition 5.12 constitute a weakly inverse correspondence in the sense that

(i) $\mathcal{R} \mathcal{P}(E \rightarrow B G)$ is weakly equivalent to $E \rightarrow B G$;

(ii) $\mathcal{P} \mathcal{R}\left(A_{\bullet} \rightarrow B_{\bullet}\right)$ is weakly equivalent to $A_{\bullet} \rightarrow B_{\bullet}$.

Theorem 5.14 establishes a "rigidification theorem", which we wish to state separately.

Theorem 5.15 Given a homotopy action of $\Omega Y$ on $X$, represented by $\pi: A_{\bullet} \rightarrow B_{\bullet}$, there is a topological group $G$ with $B G \simeq Y$ and a space $X^{\prime} \simeq X$ together with a (strict) action of $G$ on $X^{\prime}$ such that the simplicial map $\pi$ is weakly equivalent to the simplicial map Bar $\left(X^{\prime}, G\right) \rightarrow B_{\bullet}(G)$.

The proof of Theorem 5.14 will require some technical preparation.

Definition 5.16 If $A_{\bullet}$ is a simplicial space, the simplicial path space on $A_{\bullet}$, denoted $P A_{\bullet}$, is the simplicial space defined by $P A_{n}=A_{n+1}$ with face maps $d_{i}:=d_{i+1}$ and degeneracy maps $s_{i}:=s_{i+1}$. 
Observation 5.17 Let $A_{\bullet}$ be a simplicial space and let $A_{0}$ denote the constant simplicial space. There are simplicial maps $\iota: A_{0} \rightarrow A_{\bullet}$ and $\rho: P A \bullet \rightarrow A_{0}$ defined on level $n$ via the maps $[n+1] \rightarrow[0]$ and $[0] \hookrightarrow[n](0 \mapsto 0)$, respectively. $P A \bullet$ is simplicially homotopy equivalent to the constant simplicial space $A_{0}$; in particular, $\left|P A_{\bullet}\right| \simeq A_{0}$. In addition, the face map $d_{0}: A_{n+1} \rightarrow A_{n}$ defines a simplicial map $P A \bullet \rightarrow A \bullet$.

In addition, we will need the following result.

Lemma 5.18 Let $\pi: A_{\bullet} \rightarrow B \bullet$ be a homotopy action. Then for each $n \geq 0$, the square

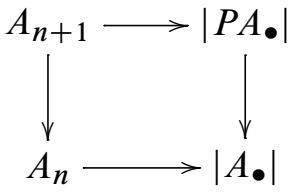

is homotopy cartesian.

Proof From the axioms of a homotopy action, there is a commutative square with horizontal maps homotopy equivalences

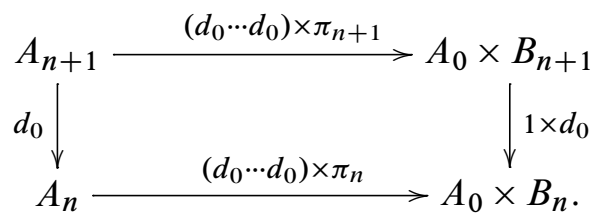

Since $B_{\bullet}$ is a reduced Segal space, by $[20,1.6]$, for each $k \geq 0$, the square

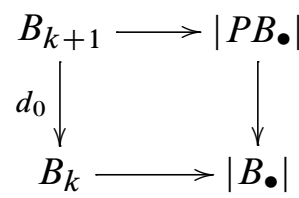

is homotopy cartesian.

Thus, the homotopy fiber of $d_{0}: B_{n+1} \rightarrow B_{n}$ is (canonically) equivalent to $B_{1}$. The homotopy fiber of $d_{0}: A_{n+1} \rightarrow A_{n}$ is therefore homotopy equivalent to $B_{1}$, which is also the homotopy fiber of $|P A \bullet| \rightarrow|A \bullet|$. It follows that the square

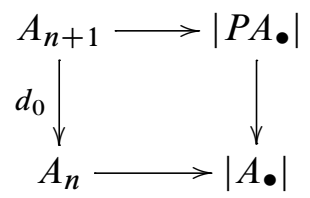

is homotopy cartesian. 
Proof of Theorem 5.14 (i) Given, without loss of generality, a fibration sequence $X \rightarrow X / G \rightarrow B G$, the map hPow॰ $(X \rightarrow X / G) \rightarrow$ hPow $_{\bullet}(* \rightarrow B G)$ obtained just as in Theorem 5.7 has $X$ as a homotopy fiber in each level. Since $\left|h \operatorname{Pow}_{\bullet}(X \rightarrow X / G)\right| \simeq$ $X / G$ and $\mid h$ Pow $_{\bullet}(* \rightarrow B G) \mid \simeq B G$, the map $\mid h$ Pow $_{\bullet}(X \rightarrow X / G)|\rightarrow| h$ Pow $_{\bullet}(* \rightarrow B G) \mid$ is equivalent to $X / G \rightarrow B G$.

(ii) Given a homotopy action $\pi: A_{\bullet} \rightarrow B_{\bullet}, B_{\bullet}$ is a reduced Segal space, and thus by [20, Proposition 1.6], for each $k \geq 0$, the following square is homotopy cartesian:

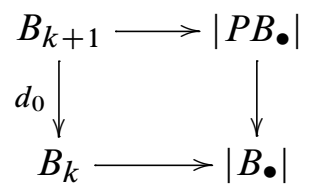

By Lemma 5.18, the same holds for $A_{\bullet}$, ie for each $k \geq 0$, the square

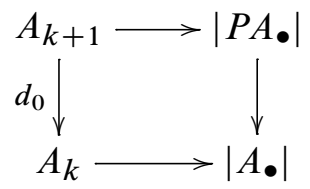

is homotopy cartesian. We construct a map $A_{\bullet} \rightarrow h P w_{\bullet}\left(\left|P A_{\bullet}\right| \rightarrow\left|A_{\bullet}\right|\right)$ by induction on $n$. For $n=0$, the map $A_{0} \rightarrow\left|P A_{\bullet}\right|$ is the realization of $\iota: A_{0} \rightarrow P A \bullet$ defined in Observation 5.17. For $n=1$, consider the commutative square

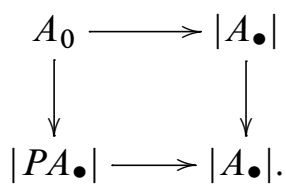

Since (2) is homotopy cartesian for $k=0$, the map $A_{1} \rightarrow A_{0} \times{ }_{\left|A_{\bullet}\right|}^{h}\left|P A_{\bullet}\right|$ is a homotopy equivalence, and the map $A_{1} \rightarrow h \operatorname{Pow}_{1}\left(\left|P A_{\bullet}\right| \rightarrow\left|A_{\bullet}\right|\right)$ is obtained by composing the last map with $A_{0} \times_{\left|A_{\bullet}\right|}^{h}\left|P A_{\bullet}\right| \rightarrow\left|P A_{\bullet}\right| \times_{\left|A_{\bullet}\right|}^{h}\left|P A_{\bullet}\right|$ induced by (3). Let us define the map for $n+1$ : the square (2) with index $n$ is homotopy cartesian, and thus there is a homotopy equivalence $A_{n+1} \rightarrow A_{n} \times_{\left|A_{\bullet}\right|}^{h}\left|P A_{\bullet}\right|$. Using the map $A_{n} \rightarrow h \operatorname{Pow}_{n}\left(\left|P A_{\bullet}\right| \rightarrow\left|A_{\bullet}\right|\right)$ that was defined, we get a natural homotopy equivalence $A_{n+1} \rightarrow$ hPow $_{\bullet}\left(\left|P A_{\bullet}\right| \rightarrow\left|A_{\bullet}\right|\right)$. It is clear from the construction that one gets a simplicial map $A_{\bullet} \rightarrow h \operatorname{Pow}_{\bullet}\left(\left|P A_{\bullet}\right| \rightarrow\left|A_{\bullet}\right|\right)$. Similarly, there is a simplicial map $B_{\bullet} \rightarrow$ hPow $_{\bullet}\left(\left|P B_{\bullet}\right| \rightarrow\left|B_{\bullet}\right|\right)$. The zigzag of commutative squares

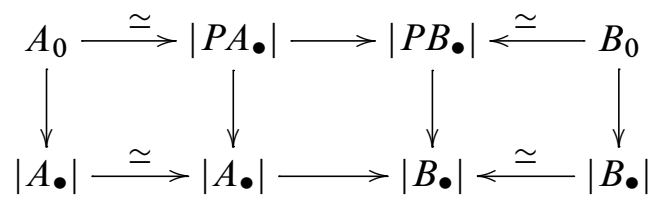


induces a zigzag of commutative simplicial squares

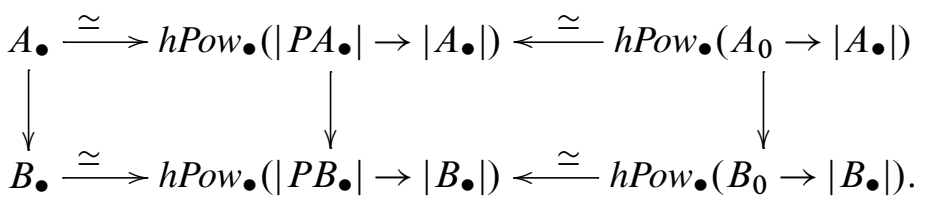

Note that by Proposition 4.1, there is also a square

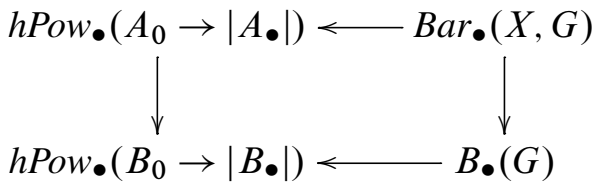

for a topological group $G$ with $B G \simeq\left|B_{\bullet}\right|$.

\section{An invariant characterization of normality}

Theorem 1.3 characterizes homotopy normal maps of discrete groups in terms of a simplicial group, equivariantly equivalent to the bar construction. By analogy, the mere fact that the homotopy bar construction $\operatorname{Bar}_{\bullet}(\Omega Y, \Omega X)$ is simplicially equivalent to a simplicial loop space $\Gamma_{\bullet}$ with $\Gamma_{0} \simeq \Omega Y$, is a necessary but not sufficient condition for a loop map $\Omega f: \Omega X \rightarrow \Omega Y$ to be homotopy normal.

In both simplicial spaces $\operatorname{Bar}_{\bullet}(\Omega Y, \Omega X)$ and $Q_{\bullet}$ (see Notation 4.6), the map $s_{n-1} \cdots s_{0}$ is a loop map, therefore it induces a homotopy action of $\Omega Y$ on $Q_{n}$ and $\operatorname{Bar}_{n}(\Omega Y, \Omega X)$ (see Corollary 5.8).

We begin with the following.

Proposition 6.1 Let $\Omega f: \Omega X \rightarrow \Omega Y$ be a homotopy normal map and $Q$. its corresponding simplicial loop space. For each $n$, the homotopy actions induced by the loop maps $Q_{0} \rightarrow Q_{n}$ and $\Omega Y \rightarrow \operatorname{Bar}_{n}(\Omega Y, \Omega X)$ are equivalent via the map $\eta: Q_{\bullet} \rightarrow \operatorname{Bar}_{\bullet}(\Omega Y, \Omega X)$, defined in Notation 4.6.

Proof We do only the case $n=1$ since other cases are similar. Write $\sigma:=$ $s_{0}: Q_{0} \rightarrow Q_{1}$ and $s:=s_{0}: \operatorname{Bar}_{0}(\Omega Y, \Omega X) \rightarrow \operatorname{Bar}_{1}(\Omega Y, \Omega X)$. The simplicial equivalence $\eta: Q_{\bullet} \rightarrow \operatorname{Bar}_{\bullet}(\Omega Y, \Omega X)$ induces a commutative square with vertical arrows being homotopy equivalences, and with the left vertical arrow being a loop map

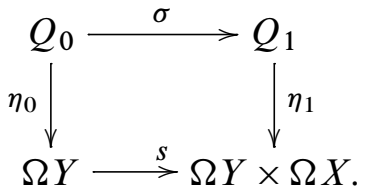


Finding the dashed arrow

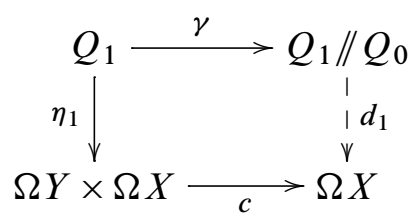

will end the proof since the first and second homotopy actions are built out of homotopy powers of $\gamma$ and $c$, respectively. Both $\sigma$ and $s$ have (spaces of the homotopy type of) loop spaces as their homotopy fiber, and the Puppe-Nomura sequence will provide the dashed arrow, once we show that the equivalence between the homotopy fibers $F:=\operatorname{hfib}(\sigma) \rightarrow \mathrm{hfib}(s) \simeq \Omega^{2} X$ is a loop map. To prove the last statement we use the path-space to model the homotopy fiber. On the one hand, we have the pullback square

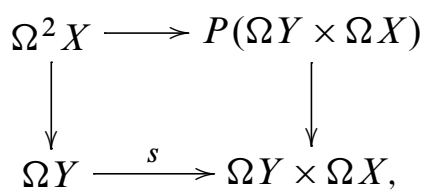

and on the other hand, in the pullback square

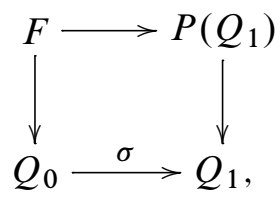

all maps are of the homotopy type of loop maps. The map $F \rightarrow \Omega^{2} X$ is the universal map to the pullback $\Omega^{2} X$, obtained from the diagram

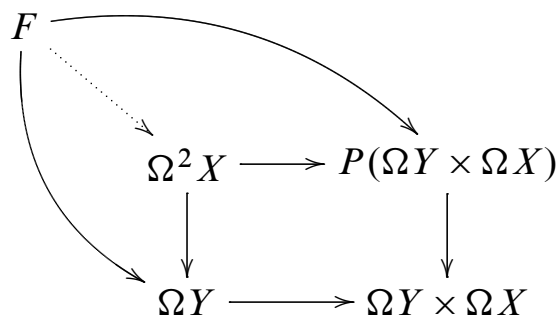

where the curved maps are $F \rightarrow Q_{0} \rightarrow \Omega Y$ and $F \rightarrow P\left(Q_{1}\right) \rightarrow P(\Omega Y \times \Omega X)$; these maps are (of the homotopy type of) loop maps, and thus the map they induce $F \rightarrow \Omega^{2} X$ is itself (of the homotopy type of) a loop map.

As we have just seen, the loop maps $s_{n-1} \cdots s_{0}: Q_{0} \rightarrow Q_{n}$ ( $n=0$ understood as the identity map) induce homotopy actions of $Q_{0}$ on $Q_{n}$. We can pack all the maps 
into one simplicial map $Q_{0} \rightarrow Q_{\bullet}$, which will then induce a simplicial object in the category of homotopy actions. Recalling Definition 5.5, this is a homotopy action of $Q_{0}$ on $Q_{\bullet}$. Similarly, one has a homotopy action of $\Omega Y$ on $\operatorname{Bar}_{\bullet}(\Omega Y, \Omega X)$ and the loop space equivalence $Q_{0} \simeq \Omega Y$ makes the first homotopy action into one of $\Omega Y$ on $Q$ • (see Corollary 5.8). Note that any simplicial loop space $\Gamma_{\bullet}$ with $\Gamma_{0} \simeq \Omega Y$ could play the role of $Q \bullet$ in defining these homotopy actions.

Given a loop map $\Omega f: \Omega X \rightarrow \Omega Y$ and a simplicial loop space $\Gamma_{\bullet}$ with $\Gamma_{0} \simeq \Omega Y$, we call the actions above the canonical homotopy actions of $\Omega Y$ on $\Gamma_{\bullet}$ and $\operatorname{Bar}_{\bullet}(\Omega Y, \Omega X)$. The additional condition for a characterization of normality is that the two are equivalent. We can now restate and prove Theorem A.

Theorem A A loop map $\Omega f: \Omega X \rightarrow \Omega Y$ is homotopy normal if and only if there exist a simplicial loop space $\Gamma_{\bullet}$ with $\Gamma_{0} \simeq \Omega Y$ (as loop spaces), and such that the canonical homotopy actions of $\Omega Y$ on $\Gamma_{\bullet}$ and on $\operatorname{Bar}_{\bullet}(\Omega Y, \Omega X)$ (as above) are weakly equivalent.

Remark 6.2 The weak equivalence of homotopy actions above implies, in particular, the equivalence of simplicial spaces $\operatorname{Bar}_{\bullet}(\Omega Y, \Omega X)$ and $\Gamma_{\bullet}$.

Proof Assume $\Omega f$ is homotopy normal. We have a commutative square of simplicial spaces

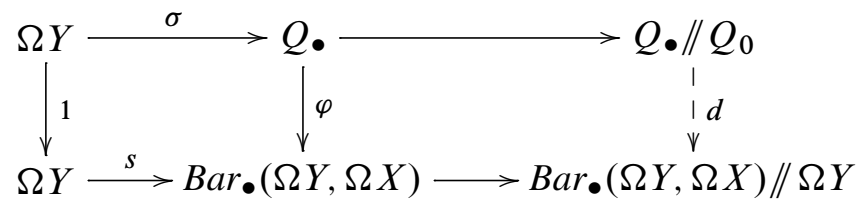

with $\varphi$ the simplicial equivalence of Theorem 4.5; the dashed arrow $d$ with $d_{1}$ (of Proposition 6.1) as its first component, and the analogous $d_{n}$ as its $n$-th component. This gives the desired equivalence of the canonical actions.

Conversely, if we have a zigzag of equivalent homotopy actions (see Definition 5.5), then taking the homotopy quotient of each homotopy action, we get a zigzag of simplicial spaces

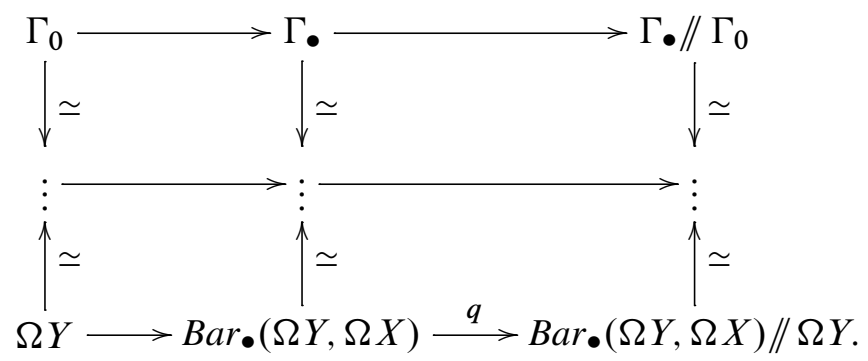


The map $q$ in the bottom row is in fact $\pi: \operatorname{Bar}_{\bullet}(\Omega Y, \Omega X) \rightarrow \operatorname{Bar}_{\bullet}(*, \Omega X)$, and upon realization we have a zigzag of equivalent principal fibrations

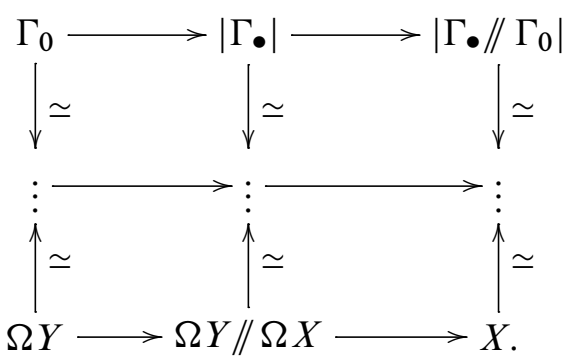

The operation of taking loops commutes with that of realization, and hence $\left|\Gamma_{\bullet}\right| \simeq \Omega W$ for some connected space $W$. The map $\Gamma_{0} \rightarrow\left|\Gamma_{\bullet}\right|$ is the realization of a simplicial loop map $\Gamma_{0} \rightarrow \Gamma_{\bullet}$, hence a loop map itself, and delooping it gives the desired extension $Y \rightarrow W$.

As an application of Theorem A we will show that homotopy normal maps are preserved by HM functors.

Let $A \bullet \rightarrow B \bullet$ be a homotopy action. From Proposition 5.13 (b), it follows that there is a homotopy fibration sequence $A_{0} \stackrel{\sigma}{\rightarrow}\left|A_{\bullet}\right| \rightarrow\left|B_{\bullet}\right|$, where $\sigma$ is the realization of the simplicial map $A_{0} \rightarrow A_{\bullet}$ that has as $n$-th component the map $s_{n-1} \cdots s_{0}$. Since $B_{\bullet}$ is a reduced Segal space, $\Omega\left|B_{\bullet}\right| \simeq B_{1}$. We denote by $\psi: B_{1} \rightarrow A_{0}$ the canonical map from the homotopy fiber of $\sigma: A_{0} \rightarrow\left|A_{\bullet}\right|$ to $A_{0}$ and endow $A_{0}$ with a basepoint via $\psi$. Denote by $i: B_{1} \rightarrow A_{0} \times B_{1}$ the natural inclusion. We shall need the following technical lemma.

Lemma 6.3 For any choice of homotopy inverse $e: A_{0} \times B_{1} \rightarrow A_{1}$ for the map $d_{1} \times \pi_{1}: A_{1} \rightarrow A_{0} \times B_{1}$, the composite

$$
B_{1} \stackrel{i}{\rightarrow} A_{0} \times B_{1} \stackrel{e}{\rightarrow} A_{1} \stackrel{d_{0}}{\rightarrow} A_{0}
$$

is homotopic to $\psi$.

Proof The following square is homotopy commutative:

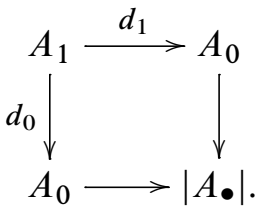


We thus obtain a homotopy commutative diagram of solid arrows

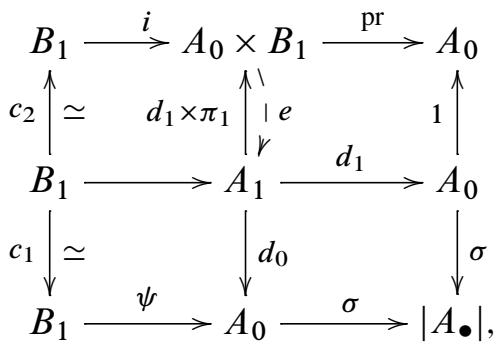

where the map $B_{1} \rightarrow A_{1}$ is the canonical map from the homotopy fiber, the map $c_{1}$ is the comparison map between the homotopy fibers of $d_{1}$ and $\sigma$, which is a homotopy equivalence, and the map $c_{2}$ is the comparison map between the homotopy fibers of $d_{1}$ and $\mathrm{pr}$, which is again a homotopy equivalence. The lemma now follows from inverting $c_{2}$.

Theorem 6.4 Let $\Omega f: \Omega X \rightarrow \Omega Y$ be a loop map and L:Top $\rightarrow$ Top an HM functor. Then the map $L \operatorname{Bar}_{\bullet}(\Omega Y, \Omega X) \rightarrow L \operatorname{Bar}_{\bullet}(*, \Omega X)$ is weakly equivalent to $\operatorname{Bar}_{\bullet}(L \Omega Y, L \Omega X) \rightarrow \operatorname{Bar}_{\bullet}(*, L \Omega X)$ where the latter is induced from $L \Omega f$.

Proof Since $L \Omega Y \rightarrow\left|L \operatorname{Bar}_{\bullet}(\Omega Y, \Omega X)\right| \rightarrow\left|L \operatorname{Bar}_{\bullet}(*, \Omega X)\right|$ is the realization of a simplicial fibration sequence, it is a homotopy fibration sequence, and since $\left|L \operatorname{Bar}_{\bullet}(*, \Omega X)\right| \simeq B(L \Omega X)\left(L \operatorname{Bar}_{\bullet}(*, \Omega X)\right.$ is a reduced Segal space for $\left.L \Omega X\right)$, there is a map $\varphi: L \Omega X \rightarrow L \Omega Y$, which is the map from the homotopy fiber of $L \Omega Y \rightarrow\left|L \operatorname{Bar}_{\bullet}(\Omega Y, \Omega X)\right|$ to $L \Omega Y$.

Abbreviate $A_{\bullet}:=\operatorname{Bar}_{\bullet}(\Omega Y, \Omega X)$ and $B_{\bullet}:=\operatorname{Bar}_{\bullet}(*, \Omega X)$. If $e: A_{0} \times B_{1} \rightarrow A_{1}$ is a homotopy inverse to $d_{1} \times \pi_{1}$, then $L e$ is a homotopy inverse for $L\left(d_{1} \times \pi_{1}\right)$, which is equivalent to $L\left(d_{1}\right) \times L\left(\pi_{1}\right)$. By Lemma 6.3, $\Omega f$ is homotopic to the composite

$$
B_{1} \stackrel{i}{\rightarrow} A_{0} \times B_{1} \stackrel{e}{\rightarrow} A_{1} \stackrel{d_{0}}{\rightarrow} A_{0},
$$

and so $L \Omega f$ is homotopic to the composition $L d_{0} \circ L e \circ L i$. The last composite is homotopic to the composite

$$
L B_{1} \hookrightarrow L A_{0} \times L B_{1} \stackrel{\text { Leow }}{\longrightarrow} L A_{1} \stackrel{L d_{0}}{\longrightarrow} A_{0}
$$

(where $w$ is some homotopy inverse for $L\left(A_{0} \times B_{1}\right) \rightarrow L A_{0} \times L B_{1}$ ), which is in turn homotopic to $\varphi$ by Lemma 6.3 ( $L e \circ w$ is a homotopy inverse for $L d_{1} \times L \pi_{1}$ ). It follows that $L \Omega f$ is equivalent to $\varphi$. Thus, the map $L \Omega Y \rightarrow|L \operatorname{Bar} \bullet(\Omega Y, \Omega X)|$ is equivalent to $L \Omega Y \rightarrow L \Omega Y / / L \Omega X$ and using Proposition 4.1 and Theorem 5.14, we deduce that $L \operatorname{Bar}_{\bullet}(\Omega Y, \Omega X) \rightarrow L \operatorname{Bar}(*, \Omega X)$ is weakly equivalent to $\operatorname{Bar}_{\bullet}(L \Omega Y, L \Omega X) \rightarrow$ $\operatorname{Bar}_{\bullet}(*, L \Omega X)$. 
Let us rephrase Theorem 6.4. Given a loop map $\Omega f$ and an HM functor $L$, there are two homotopy actions: the first is given by applying $L$ to the homotopy action induced by $\Omega f$, and the second is the homotopy action induced from $L \Omega f$. The theorem then says that the two are weakly equivalent. We note that if we are given a homotopy action of a loop space on a simplicial space, in which the homotopy actions in each level are induced by loop maps, an analogous statement holds.

Using the machinery of reduced Segal spaces, one can easily see that applying an HM functor to a simplicial loop space in every level yields a simplicial space simplicially equivalent to a simplicial loop space.

Thus, we now know all the ingredients used in Theorem A are invariant under HM functors and we deduce Theorem B (which we restate for convenience).

Theorem B Let $\Omega f: \Omega X \rightarrow \Omega Y$ be a homotopy normal map. If $L:$ Top $\rightarrow$ Top is an $H M$ functor, then $L(\Omega f): L \Omega X \rightarrow L \Omega Y$ is a homotopy normal map.

Let us demonstrate a use of Theorem B by applying it to prove Theorem $\mathrm{C}$ (which we restate).

Theorem C Let $p: E \rightarrow B$ be a principal fibration with $B$ connected, $f: X \rightarrow Y$ a map of pointed connected spaces and $L_{\Sigma f}$ the localization with respect to its suspension. Then $L_{\Sigma f} E \rightarrow L_{\Sigma f} B$ is equivalent to a principal fibration.

Remark 6.5 Note that if $G$ is the structure group of $E \rightarrow B, L_{\Sigma f} G$ need not be the structure group of $L_{\Sigma f} E \rightarrow L_{\Sigma f} B$.

Proof of Theorem C Note that $\Omega E \rightarrow \Omega B$ is homotopy normal. So $L_{f} \Omega E \rightarrow L_{f} \Omega B$ is homotopy normal. Since for any pointed space $A$ there is a natural equivalence $L_{f} \Omega A \simeq \Omega L_{\Sigma f} A$, we get that $\Omega L_{\Sigma f} E \rightarrow \Omega L_{\Sigma f} B$ is homotopy normal and thus $L_{\Sigma f} E \rightarrow L_{\Sigma f} B$ is a homotopy principal fibration.

\section{Higher normality}

As mentioned in Example 2, any double loop map with simply connected underlying spaces is automatically homotopy normal. However, in the case of a double loop map, it is more natural to ask when the homotopy quotient admits a natural double loop space structure. 
Definition 7.1 A 0-homotopy normal map is a pointed map which admits a structure of a (homotopy) principal fibration of connected spaces. For $k \geq 1$, call a $k$-fold loop map $\Omega^{k} f: \Omega^{k} X \rightarrow \Omega^{k} Y k$-homotopy normal if $f$ is 0 -homotopy normal.

Thus, if a $k$-fold loop map $\Omega^{k} f$ is $k$-homotopy normal, the homotopy quotient $\Omega^{k} Y / / \Omega^{k} X$ (which is always a $(k-1)$-fold loop space) admits a structure of a $k$-fold loop space in a natural way.

Remark 7.2 One may wonder about the definition of " $\infty$-homotopy normality". However, any infinite loop map $X \rightarrow Y$ induces a principal fibration sequence of infinite loop spaces $X \rightarrow Y \rightarrow Y / / X$. Thus any infinite loop map is " $\infty$-normal" in the naive sense. This is a reflection of the fact that any inclusion map of abelian (topological) groups is the inclusion of a normal subgroup.

We begin with an extension of Theorem A.

Theorem 7.3 A $k$-fold loop map $\Omega^{k} f: \Omega^{k} X \rightarrow \Omega^{k} Y$ is $k$-homotopy normal if and only if there exists a $k$-fold simplicial loop space $\Gamma_{\bullet}$ with $\Gamma_{0} \simeq \Omega^{k} Y$, and such that the canonical homotopy actions of $\Omega^{k} Y$ on $\operatorname{Bar} \bullet\left(\Omega^{k} Y, \Omega^{k} X\right)$ and $\Gamma_{\bullet}$ are naturally equivalent.

Proof This is analogous to the proof of Theorem C. If $\Omega^{k} f$ is $k$-homotopy normal, then $\Omega f$ is homotopy normal, and looping down its extension $Y \rightarrow W k$ times gives a $k$-fold loop map equivalent to $\Omega^{k} Y \rightarrow \Omega^{k} Y / / \Omega^{k} X$. Taking the (homotopy) power of that map gives the desired $k$-fold loop space. Conversely, such a $k$-fold loop space gives a (homotopy) principal fibration sequence of $k$-fold loop spaces $\Omega^{k} X \rightarrow \Omega^{k} Y \rightarrow\left|\Gamma_{\bullet}\right|$, equivalent to the Borel construction, providing the $k$-homotopy normality required.

We wish to use the same methods as before to prove invariance of $k$-homotopy normal maps under $H M$ functors. For that, we need that $k$-fold loop spaces are invariant under these functors. A slight generalization of reduced Segal spaces is the tool needed.

Definition 7.4 Let $k$ be a positive integer. A $k$-simplicial space is a functor

$$
\Delta^{\mathrm{op}} \times \cdots \times \Delta^{\mathrm{op}} \rightarrow \text { Top } \quad(k \text { times }) .
$$

The following is taken from Balteanu et al [1]. 


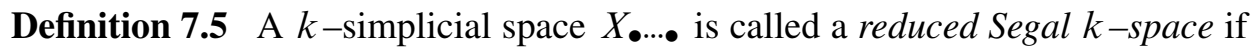

(1) $X_{0, \ldots, 0} \simeq *$;

(2) the Segal maps induce homotopy equivalences $X_{p_{1}, \ldots, p_{k}} \stackrel{\simeq}{\rightarrow}\left(X_{1, \ldots, 1}\right)^{p_{1} \cdots p_{k}}$;

(3) the monoid $\pi_{0}\left(X_{1, \ldots, 1}\right)$ admits inverses (ie is a group).

Building on Segal's delooping machine, the characterization of $k$-fold loop spaces takes the following form.

Theorem 7.6 A space $X$ is of the homotopy type of a $k$-fold loop space if and only if there exist a reduced Segal $k$-space $X_{\bullet}, \ldots, \bullet$ with $X_{1, \ldots, 1} \simeq X$.

Corollary 7.7 Homotopy monoidal endofunctors of spaces preserve $k$-fold loop spaces.

Using exactly the same arguments of Theorem C, Theorem 7.3 implies that $L$ preserves higher homotopy normality.

Theorem 7.8 If $\Omega^{k} f: \Omega^{k} X \rightarrow \Omega^{k} Y$ is $k$-homotopy normal and L: Top $\rightarrow$ Top an HM functor, then $L\left(\Omega^{k} f\right)$ is $k$-homotopy normal.

\section{References}

[1] C Balteanu, Z Fiedorowicz, R Schwänzl, R Vogt, Iterated monoidal categories, Adv. Math. 176 (2003) 277-349 MR1982884

[2] A K Bousfield, Unstable localization and periodicity, from: "Algebraic topology: new trends in localization and periodicity (Sant Feliu de Guíxols, 1994)", (C Broto, C Casacuberta, G Mislin, editors), Progr. Math. 136, Birkhäuser, Basel (1996) 33-50 MR1397720

[3] R Brown, P J Higgins, R Sivera, Nonabelian algebraic topology: Filtered spaces, crossed complexes, cubical higher homotopy groupoids, EMS Tracts in Math. 15, European Math. Soc. (2010) With contributions by CD Wensley and S V Soloviev

[4] E Dror, W G Dwyer, D M Kan, Equivariant maps which are self homotopy equivalences, Proc. Amer. Math. Soc. 80 (1980) 670-672 MR587952

[5] W G Dwyer, E D Farjoun, Localization and cellularization of principal fibrations, from: "Alpine perspectives on algebraic topology", (C Ausoni, K Hess, J Scherer, editors), Contemp. Math. 504, Amer. Math. Soc. (2009) 117-124 MR2581908

[6] W G Dwyer, C W Wilkerson, Homotopy fixed-point methods for Lie groups and finite loop spaces, Ann. of Math. 139 (1994) 395-442 MR1274096 
[7] E D Farjoun, Cellular spaces, null spaces and homotopy localization, Lecture Notes in Math. 1622, Springer, Berlin (1996) MR1392221

[8] E D Farjoun, K Hess, Normal and conormal maps in homotopy theory arXiv: 1011.5597

[9] E D Farjoun, Y Segev, Crossed modules as homotopy normal maps, Topology Appl. 157 (2010) 359-368 MR2563286

[10] T G Goodwillie, Calculus II: Analytic functors, K-Theory 5 (1991/92) 295-332 MR1162445

[11] D H Gottlieb, The total space of universal fibrations, Pacific J. Math. 46 (1973) 415417 MR0331384

[12] D M Kan, On homotopy theory and c.s.s. groups, Ann. of Math. 68 (1958) 38-53 MR0111033

[13] J-L Loday, Spaces with finitely many nontrivial homotopy groups, J. Pure Appl. Algebra 24 (1982) 179-202 MR651845

[14] J P May, Classifying spaces and fibrations, Mem. Amer. Math. Soc. 1, no. 155, Amer. Math. Soc. (1975) MR0370579

[15] J Milnor, Construction of universal bundles. I, II, Ann. of Math. (2) 63 (1956) 272-284, 430-436 MR0077122

[16] Y Nomura, On mapping sequences, Nagoya Math. J. 17 (1960) 111-145 MR0132545

[17] R A Nowlan, $A_{n}$-actions on fibre spaces, Indiana Univ. Math. J. 21 (1972) 285-313 MR0288767

[18] V Puppe, A remark on “homotopy fibrations”, Manuscripta Math. 12 (1974) 113-120 MR0365556

[19] C Rezk, A model for the homotopy theory of homotopy theory, Trans. Amer. Math. Soc. 353 (2001) 973-1007 MR1804411

[20] G Segal, Categories and cohomology theories, Topology 13 (1974) 293-312 MR0353298

[21] J Stasheff, “Parallel” transport-revisited arXiv:1111.5495

[22] J Stasheff, H-spaces from a homotopy point of view, Lecture Notes in Math. 161, Springer, Berlin (1970) MR0270372

[23] J H C Whitehead, Combinatorial homotopy. II, Bull. Amer. Math. Soc. 55 (1949) 453-496 MR0030760

Einstein Institute of Mathematics, Hebrew University of Jerusalem

Giv'at Ram, Jerusalem 91904, Israel

matan.prezma@mail.huji.ac.il

Received: 31 May $2011 \quad$ Revised: 17 November 2011 